\title{
Cesáreas en el Instituto Materno-Infantil de Marzo a Agosto de 1981
}

\author{
Universidad Nacional de Colombia \\ Departamento de Ginecología y Obstetricia
}

Dres.: Nelly R. de Gómez ${ }^{1}$ Víctor A. Toro ${ }^{2}$ Víctor M. Rodríguez ${ }^{3}$

Coordinador: Dr. Rodrigo Díaz LI. Docente Gineco-Obstetricia

\section{INTRODUCCION}

La operación cesárea es un procedimiento que ha venido incrementándose en el mundo debido a la aparición de nuevas indicaciones, mejoramiento en las técnicas $\mathrm{y}$ medios quirúrgicos, progresos en anestesia y en la valoración del medio ambiente fetal $(1,2,3,4,5,6,7,8,9$, 10). Por tal motivo se realizó el presente trabajo descriptivo-prospectivo en el Instituto Materno Infantil de Bogotá, para hacer un aporte respecto a indicaciones, conductas, morbi-mortalidad y otras complicaciones, tanto maternas como fetales, que se derivan de este procedimientn.

En el país no se ha realizado un estudio como el propuesto y solo se han enfocado diferentes aspectos del tema; encontrándose en la literatura estudios de morbi-mortalidad materna y fetal (11,

1. $R_{3}$ Gineco-Obstetricia

2. $R_{3}$ Gineco-Obstetricia

3. $R_{2}$ Gineco-Obstetricia
12 , 13), parto por vía vaginal luego de cesárea $(14,15,16,17,18)$, aportes a la técnica de cesárea extraperitoneal $(19$. $20,21)$ y conducta obstétrica en pacientes con cesárea previa $(22,23,24)$. En el Instituto Materno Infantil el último trabajo sobre el tema data del año 1967 (14).

\section{MATERIALES Y METODOS}

Se realizó un estudio prospectivo de los casos de aito riesgo atendidos entre el 1 de marzo y el 31 de agosto de 1981 por los diferentes grupos de Obstetricia que funcionan en el Departamento; cada grupo obstétrico está formado por un jefe de turno, médicos adjuntos, médicos residentes e internos.

Para recolectar los datos se elaboró un formulario con 32 variables, que fue llenado en su mayor parte dentro de las 24 horas siguientes a la práctica de cesárea; solo algunos datos que se completaron al egreso de la paciente. Se reunieron 605 casos, de los cuales se 
desecharon 56 por mala elaboración en las historias, restando 549 para estudio.

La tabulación de la información se hizo manualmente y su presentación se consigna en los gráficos que posteriormente se analizan.

\section{RESULTADOS}

La frecuencia de la operación cesárea en el Instituto Materno Infantil contra el número de nacidos vivos se muestran en el cuadro No. 1.

\section{Cuadro No. 1}

\begin{tabular}{|cc|}
\hline 1978 & $6.82 \%$ \\
1979 & $6.98 \%$ \\
1980 & $7.50 \%$ \\
1981 & $12.61 \%$ \\
\hline
\end{tabular}

Gráfico No. 1

PORCENTAJE DE CESAREAS SEGUN GRUPO DE EDAD

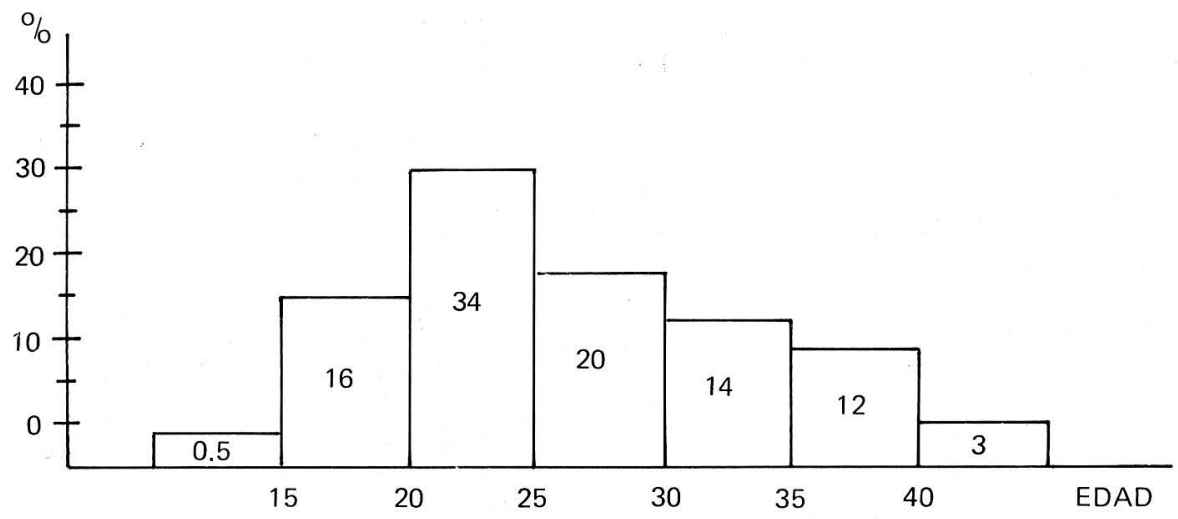

Como puede observarse, el $70.9 \%$ de cesáreas se practicó en pacientes entre los 15 y 30 años, con mayor frecuencia entre los 20 y 24 años.

La edad gestacional que ofrece la mayor frecuencia se encuentra en embarazos mayores de 35 semanas.

La mayoría de las pacientes tenían más de un embarazo cuando les fue practicada la cesárea, siendo el mayor promedio entre 1 y 3 embarazos.
Cuadro No. 2

EDAD GESTACIONAL EN CESAREAS PRACTICADAS

\begin{tabular}{|l|c|c|}
\hline Edad gestacionai & No. casos & Porcentaje \\
\hline$\leqslant 28$ semanas & 5 & $0.9 \%$ \\
$29-34 \quad "$ & 42 & 7.7 \\
$\geqslant 35 \quad "$ & 502 & 91.4 \\
\hline TOTAL & 549 & 100.0 \\
\hline
\end{tabular}


Cuadro No. 3

PARIDAD EN CESAREAS

\begin{tabular}{|c|c|c|}
\hline Paridad & No. Casos & Porcentaje \\
\hline 0 & 119 & 21.7 \\
$1-3$ & 341 & 62.1 \\
$4-6$ & 63 & 11.5 \\
$\geqslant 7$ & 26 & 4.7 \\
\hline TOTAL & 549 & 100.0 \\
\hline
\end{tabular}

En este estudio se encontró que un poco más de la mitad de las pacientes no habían asistido a control prenatal: $54 \%$.

De las 549 pacientes estudiadas, 182 tenían cesárea anterior; a 367 no se les había practicado ninguna cesárea.

Cuadro No. 4

\section{CESAREAS ANTERIORES A LA ACTUAL}

\begin{tabular}{|c|c|c|}
\hline No. Cesáreas & No. Casos & Porcentaje \\
\hline 1 & 130 & 71.5 \\
2 & 43 & 23.6 \\
3 & 9 & 4.9 \\
\hline TOTAL & 182 & 100.0 \\
\hline
\end{tabular}

Cuadro No. 5

ALTURA UTERINA AL INGRESO

\begin{tabular}{|c|c|c|}
\hline Altura uterina & No. casos & Porcentaje \\
\hline$\leqslant 26 \mathrm{cms}$. & 20 & 3.6 \\
$27-29 "$ & 101 & 18.4 \\
$30-32 "$ & 258 & 47.0 \\
$\geqslant 33 " \prime$ & 170 & 31.0 \\
\hline TOTAL & 549 & 100.0 \\
\hline
\end{tabular}

Cuadro No. 6

ESTADO DEL FETO AL INGRESO

\begin{tabular}{|l|c|c|}
\hline Estado & No. casos & Porcentaje \\
\hline Vivos & 530 & 96.5 \\
Muertos & 19 & 3.5 \\
\hline TOTAL & 549 & 100.0 \\
\hline
\end{tabular}

Cuadro No. 7

PRESENTACION FETAL AL INGRESO

\begin{tabular}{|l|c|c|}
\hline Presentación & No. casos & Porcentaje \\
\hline Cefálica & 441 & 80.3 \\
Pelvis & 85 & 15.5 \\
Hombro & 23 & 4.2 \\
\hline TOTAL & 549 & 100.0 \\
\hline
\end{tabular}

En la presentación cefálica están incluidas la variedad de vértice, cara y frente.

Cuadro No. 8

ESTADO DE LAS MEMBRANAS AMNIOTICAS AL INGRESO

\begin{tabular}{|l|c|c|}
\hline \multicolumn{1}{|c|}{ Membranas } & No. casos & Porcentaje \\
\hline Integras & 372 & 67.8 \\
Rotas $<$ 6 horas & 62 & 11.3 \\
Rotas 6 - 10 horas & 41 & 7.5 \\
Rotas 11 y más & 74 & 13.4 \\
\hline TOTAL & 549 & 100.0 \\
\hline
\end{tabular}

La mayoría de las pacientes ingresaron con membranas íntegras, solo 177 tenían membranas rotas con diferente tiempo de ruptura.

A la mayoría, $53,6 \%$ solo se les practicó $1-2$ tactos vaginales. 
Cuadro No. 9

INFECCION POST-OPERATORIA RELACIONADA CON ESTADO DE MEMBRANAS

\begin{tabular}{|l|c|c|c|c|c|}
\hline \multicolumn{2}{|l|}{$\begin{array}{l}\text { Estado de } \\
\text { las membras }\end{array}$} & $\begin{array}{l}\text { Sin } \\
\text { infección }\end{array}$ & Endometritis & $\begin{array}{l}\text { Absceso } \\
\text { de pared }\end{array}$ & Total \\
\hline \multicolumn{2}{|c|}{ Integras } & $256(67 \%)$ & $84(67 \%)$ & $32(73 \%)$ & 372 \\
\hline $\begin{array}{l}r \\
0\end{array}$ & $<6$ hrs. & $47(12 \%)$ & $11(9 \%)$ & $4(9 \%)$ & 62 \\
\cline { 2 - 6 } & $6-10^{\prime \prime}$ & $26(7 \%)$ & $10(8 \%)$ & $5(11 \%)$ & 41 \\
\cline { 2 - 6 } & $\geqslant 11 "$ & $51(14 \%)$ & $20(16 \%)$ & $3(7 \%)$ & 74 \\
\hline \multicolumn{2}{l|l}{ TOTAL } & $380(100 \%)$ & $125(100 \%)$ & $44(100 \%)$ & \\
\hline
\end{tabular}

Cuadro No. 10

TACTOS VAGINALES ANTES DE LA CESAREA

\begin{tabular}{|c|c|c|}
\hline No. Tactos vaginales & No. casos & Porcentaje \\
\hline $1-2$ & 294 & 53.6 \\
$3-5$ & 214 & 39.0 \\
$>5$ & 41 & 7.4 \\
\hline TOTAL & 549 & 100.0 \\
\hline
\end{tabular}

Cuadro No. 11

INFECCION POST-OPERATORIA RELACIONADA CON TACTO VAGINAL

\begin{tabular}{|c|c|c|c|}
\hline $\begin{array}{c}\text { Tactos } \\
\text { vaginales }\end{array}$ & $\begin{array}{c}\text { Sin } \\
\text { infección }\end{array}$ & Endometritis & Abscesos \\
\hline $1-2$ & 212 & 64 & 18 \\
$3-5$ & 143 & 50 & 21 \\
$>6$ & 25 & 11 & 5 \\
\hline TOTAL & 380 & 125 & 44 \\
\hline
\end{tabular}

La principal indicación fue la desproporción céfalo-pélvica, en un porcentaje
Cuadro No. 12

INDICACION DE LA CESAREA ACTUAL

\begin{tabular}{|l|r|r|}
\hline Indicación Cesárea & No. casos & Porcentaje \\
\hline Desproporción & 160 & 29.1 \\
feto-pélvica & 94 & 17.1 \\
Iterativez & 75 & 13.7 \\
Presentación pelvis & 42 & 7.6 \\
Sufrimiento fetal & 34 & 6.2 \\
Placenta previa & 26 & 4.7 \\
Toxemia & 20 & 3.7 \\
Situación transversa & 14 & 2.5 \\
Abruptio placentae & 84 & 15.3 \\
Otros & 549 & 100.0 \\
\hline TOTAL
\end{tabular}

de $29.1 \%$, seguida de iterativez en un $17.1 \%$.

La mayoría de las cesaréas, $62.5 \%$, fueron practicadas por médicos Residentes.

Se observa una estancia de $4-6$ días, en la mayoría de las pacientes, las estancias mayores de 10 días se debieron a complicaciones. 
Cuadro No. 13

DESPROPORCION FETO-PELVICA Vs.

PESO DEL R.N

\begin{tabular}{|l|c|c|}
\hline Peso Grm. & No. casos & Porcentaje \\
\hline$\gtrless 2.499$ & 11 & 6.9 \\
$2.500-3.499$ & 91 & 56.9 \\
$\geqslant 3.500$ & 56 & 35.0 \\
Sin dato & 2 & 1.2 \\
\hline TOTAL & 160 & 100.0. \\
\hline
\end{tabular}

Cuadro No. 14

CIRUGIAS ASOCIADAS A LAS CESAREAS

\begin{tabular}{|l|c|c|}
\hline \multicolumn{1}{|c|}{ Cirugías } & No. casos & Porcentaje \\
\hline Ligadura de trompas & 80 & 97.6 \\
Ooforectomía & 1 & 1.2 \\
Histerectomía & 1 & 1.2 \\
\hline TOTAL & 82 & 100.0 \\
\hline
\end{tabular}

Cuadro No. 15

TECNICA QUIRURGICA EMPLEADA EN LA CESAREA

\begin{tabular}{|l|c|c|}
\hline Técnica & No. casos & Porcentaje \\
\hline Transperitoneal & 542 & 98.7 \\
Extraperitoneal & 6 & 1.1 \\
Corporal & 1 & 0.2 \\
\hline TOTAL & 549 & 100.0 \\
\hline
\end{tabular}

Cuadro No. 16

CIRUJANO QUE PRACTICO LA CESAREA

\begin{tabular}{|l|c|c|}
\hline Categoría & No. casos & Porcentaje \\
\hline Docente & 15 & 2.7 \\
Adjunto & 84 & 15.3 \\
Residente & 343 & 62.5 \\
Interno & 107 & 19.5 \\
\hline TOTAL & 549 & 100.0 \\
\hline
\end{tabular}

Cuadro No. 17

\section{ANESTESIA ADMINISTRADA}

\begin{tabular}{|l|r|r|}
\hline Anestesia & No. casos & Porcentaje \\
\hline General & 56 & 10.2 \\
Peridural & 454 & 82.7 \\
Raquia & 39 & 7.1 \\
\hline TOTAL & 549 & 100.0 \\
\hline
\end{tabular}

Cuadro No. 18

APGAR DEL RECIEN NACIDO

\begin{tabular}{|c|c|c|}
\hline Apgar & No. casos & Porcentaje \\
\hline 0 & 36 & 6.5 \\
$1-3$ & 40 & 7.2 \\
$4-6$ & 49 & 8.8 \\
$7-10$ & 430 & 77.5 \\
\hline TOTAL & 555 & 100.0 \\
\hline
\end{tabular}

Cuadro No. 19

PESO DEL RECIEN NACIDO

\begin{tabular}{|l|c|c|}
\hline \multicolumn{1}{|c|}{ Peso (grm) } & No. casos & Porcentaje \\
\hline$<1499$ & 20 & 3.6 \\
$1500-2499$ & 89 & 16.0 \\
$2500-3499$ & 338 & 61.0 \\
$>3500$ & 108 & 19.4 \\
\hline TOTAL & 555 & 100.0 \\
\hline
\end{tabular}

Cuadro No. 20

ENTREGA DEL RECIEN NACIDO

A LA MADRE

\begin{tabular}{|l|c|c|}
\hline Tiempo en horas & No. casos & Porcentaie \\
\hline Antes de 24 horas & 290 & 52.3 \\
Después de 24 horas & 206 & 37.1 \\
Muertos & 59 & 10.6 \\
\hline TOTAL & 555 & 100.0 \\
\hline
\end{tabular}


Cuadro No. 21

\section{COMPLICACION POST-OPERATORIA}

\begin{tabular}{|lc|}
\hline Complicación & No. casos \\
\hline Endometritis & 125 \\
Absceso de pared & 44 \\
Retención urinaria & 11 \\
Infección urinaria & 8 \\
Bronconeumonía & 8 \\
Dehiscencia histerorrafia & 5 \\
Hemorragia & 4 \\
Absceso tubo-ovárico & 3 \\
Peritonitis & 3 \\
Miometritis & 2 \\
Otros & 7 \\
\hline TOTAL & 220 \\
\hline
\end{tabular}

Cuadro No. 22

ESTANCIA POST-OPERATORIA

\begin{tabular}{|l|r|r|}
\hline No. de días & No. casas & Porcentaje \\
\hline$<4$ & 19 & 3.5 \\
$4-6$ & 319 & 58.1 \\
$7-10$ & 138 & 25.1 \\
$>10$ & 73 & 13.3 \\
\hline TOTAL & 549 & 100.0 \\
\hline
\end{tabular}

\section{DISCUSION}

Este estudio prospectivo realizado en un período de 6 meses (1981), tomó un grupo de 549 pacientes a quienes se les practicó operación cesárea para la terminación de su embarazo, con el fin de evaluar los resultados.

En 1981 se observó casi una duplicación en la proporción de cesáreas en comparación con los $3 \cdot$ años anteriores (Cuadro No. 1) y este incremento se explica porque se presentó una restricción en la capacidad locativa del |M| por el traslado de la institución a los pisos 7 y 8 del Hospital San Juan de Dios (H.S.J.D.). Se inició en ese año la remodelación total de la sede del IMI (la capacidad de camas obstétricas en el IMI antes de 1981 era de 285 y en el H.S.J.D. se dispone de 154). Esto obligó a limitar el ingreso de pacientes de bajo riesgo que antes eran atendidas en el $\mid \mathrm{MI}$, para remitirlas a otros centros, disminuyendo así el número de nacidos vivos en el IMI, con el explicable aumento de proporción de cesáreas. La distribución de la cesárea por grupos de edad (Gráfico No. 1) es comparable con esta misma distribución observada para todos los partos en general (25) y corresponde a los índices de fertilidad por edades en mujeres colombianas (26).

De las 549 pacientes, $182(33 \%)$ tenían cesárea anterior (Cuadro No. 4); la mayoría tenían una cesárea anterior a la actual y solo 9 pacientes tenían 3 cesáreas previas, lo que implica que con la actual completarían 4 intervenciones. A estas 9 pacientes se les practicó ligadura de trompas. No se encontró ningún caso de embarazadas con antecedente de 4 cesáreas.

En el cuadro No. 5 se compara la altura uterina contra el número de casos; la mayoría de las pacientes $(78 \%)$ tenían una A.U. mayor de $30 \mathrm{cms}$, las pacientes operadas con A.U. menor o igual a $26 \mathrm{cms}$, tuvieron indicaciones que urgían el procedimiento: toxemia que no respondía al tratamiento $y$ con varias inducciones fallidas (8 casos), placenta previa sangrante ( 6 casos), pacientes con 3 cesáreas anteriores $y$ en trabajo de parto (4 casos), trabajo de parto con situación transversa (2 casos).

En el cuadro No. 6 se observa que al ingreso había ruidos cardíacos fetales 
positivos en 530 casos $(96.5 \%)$ y negativos en $19(3.5 \%)$. La indicación de cesárea en pacientes con feto muerto fue: abruptio placentae (5 casos), situación transversa ( 3 casos), placenta previa (3 casos), iterativez (3 casos), variedad de frente (1 caso), desproporción feto pélvica ( 2 casos), presentación de pelvis y cesárea anterior (2 casos).

La mayoría de las pacientes $(68 \%)$ ingresaron con membranas íntegras (cuadro No. 8).

Se comparó la aparición posterior de endometritis (cuadro No. 9) entre los grupos con membranas íntegras y rotas y no se encontraron diferencias estadísticamente significativas $P<0.05$. Se esperaba que en las pacientes con membranas íntegras la endometritis postcesárea fuera menor que en los grupos con membranas rotas. El hecho de que no sea significativa la diferencia hace suponer que existen otros factores que inciden en la aparición de la infección y que pueden ser de orden locativos, institucional, contaminación, o estado nutricional de la paciente.

En la mayoría de los casos $(53.6 \%)$ se practicó de 1 a 2 tactos vaginales (cuadro No. 10), lo cual estaría indicando que la conducta que se siguió se definió en el momento de ingreso al hospital.

Es de anotar que la indicación más frecuente de la cesárea fue la desproporción feto-pélvica, en 160 casos $(29 \%)$; sin embargo, de estos solo 56 nacidos vivos tuvieron peso superior a 3.500 grm. (Cuadro No. 13) y hubo 11 casos con peso inferior a 2.500 grm. En 3 casos se confundió el tamaño fetal con embarazo gemelar y en otros la desproporción se estableció teniendo en cuenta la pelvis materna. La segunda indicación fue la iterativez en 94 casos $(17 \%)$. En el presente estudio hubo 182 pacientes con cesárea anterior, pero 88 tuvieron indicación quirúrgica diferente. La indicación quirúrgica por sufrimiento fetal que se presentó en 42 casos $(7.6 \%)$, se estableció clínicamente y no siempre por monitoría fetal. El $\mathrm{pH}$ de sangre fetal obtenida de cuero cabelludo no se practica en la Institución. Se realizaron 26 cesáreas $(4.7 \%)$ en pacientes toxémicas. Esta indicación para llevar a una paciente a cirugía puede no ser suficiente ya que esta patología por sí sola no es indicación. Seguramente existió otro factor que determinó el procedimiento pero no pudo ser tabulado por deficiencias en la historia. La ligadura de trompas se practicó en pacientes que solicitaban esta intervención. (Cuadro No. 14); ooforectomía unilateral en una paciente con quiste de ovario de gran tamaño y la histerectomía en un abruptio placentae con útero de Couvalaire.

Las cesáreas extraperitoneales se practicaron por amnionitis; corporal se practico en una paciente con hipertensión crónica con toxemia sobre-agregada y antecedente de 3 cesáreas, en el acto quirúrgico fue imposible practicar la técnica segmentaria, a esta paciente se le ligaron trompas.

Un porcentaje alto de cesáreas fueron realizadas por residentes y solo 15 casos $(2.7 \%$ ) por personal docente (Cuadro No. 16), a pesar de que la Institución tiene carácter universitario.

La anestesia de elección fue la peridural en 454 casos $(82.7 \%)$, que ofrece menor riesgo fetal y materno (Cuadro No. 17).

De los 36 fetos con APGAR 0 (Cuadro No. 18), 19 ingresaron con diagnóstico de feto muerto, los 17 restantes murieron en la Institución. El número total de índices de APGAR registrados fue de 555, ya que se presentaron 6 embarazos gemelares. De estos niños necesitaron reanimación $76(14 \%)$ procedimiento que en la mayoría se realizó en las horas de la mañana. 
Casi todos los recién nacidos pesaron más de $2.500 \mathrm{grm}$. (Cuadro No. 19), hubo 20 niños $(3.6 \%)$ con peso menor de $1.500 \mathrm{grm}$. que correspondieron a las pacientes con altura uterina $\leqslant 26 \mathrm{~cm}$.

De los 59 niños muertos (Cuadro No. 20), la mayoría nacieron con APGAR de $1-3$ (11 casos); si tenemos en cuenta que hubo 40 niños con APGAR entre $1-3$ y 29 sobrevivieron $(72.5 \%)$, hay que reconocer que hubo un manejo adecuado de los recién nacidos.

El $78 \%$ de los recién nacidos tuvieron un APGAR mayor de 7 y el $53 \%$ fueron entregados a la madre antes de las 24 horas.

La morbilidad materna (Cuadro No. 21), está dada por la infección en sus diferentes modalidades principalmente endometritis y abscesos de pared ( 76 . $8 \%$ ). Hubo otras complicaciones como absceso tubo-ovárico, peritonitis, miometritis, infección urinaria $y$ bronconeumonía.

Se administraron antibióticos a 286 pacientes $(52 \%)$ en el período postoperatorio, por diferentes indicaciones, algunas de las cuales no aparecen muy claras en la historia; 80 de ellas las recibieron desde antes de la cirugía, sin existir signos clínicos de infección; en ningún caso se utilizaron asociaciones antibióticas.

A dos pacientes se les practicó histerectomía por miometritis y de ellas una murió por sepsis. La mortalidad materna fue de $0.18 \%$ (una paciente), debida a sepsis y con infección amniótica al ingreso. Como puede observarse esta tasa es baja con relación a otros estudios publicados $(4,5,7,11)$.

La estancia promedio de las pacientes (Cuadro No. 22), osciló entre 4 y 6 días; sin embargo, hubo 73 casos en que las pacientes permanecieron más de 10 días, la mayoría por complicaciones, siendo de éstas la infección la más importante.

\section{RESUMEN}

Se analizaron 549 historias de pacientes a quienes se les practicó operación cesárea en el IMI entre marzo y agosto de 1981, encontrándose un aumento.en la proporción de cesáreas que llegó al $12.6 \%$.

La desproporción feto pélvica continúa como primera indicación $(29 \%)$, seguida de la iterativez y aparece la toxemia como indicación en un porcentaje apreciable $(4.7 \%)$.

La técnica más usada fue la transperitoneal $(98 \%)$ y la anestesia preferida la peridural $(82 \%)$, actuando el personal en entrenamiento como cirujano en un alto número de las cesáreas $(82 \%)$.

Las complicaciones post-operatorias fueron de índole infecciosa en la mayoría de las veces, siendo las más frecuentes la endometritis y el absceso de pared; sin embargo, no se encontró relación entre esta patología y el estado de membranas rotas al ingreso. Hubo una muerte materna por sepsis $(0.18 \%)$. El promedio de estancia hospitalaria fue de 5 días.

La mayoría de los recién nacidos tuvo peso entre 2.500 y $3.500 \mathrm{grm}$. y APGAR de 7 a 10. Ocurrieron 23 muertes de recién nacidos y 17 casos de fetos muertos durante el trabajo de parto; en 19 casos se encontraron ruidos fetales negativos al ingreso, siendo en ellos la indicación de la cirugía de índole exclusivamente materna. 


\section{CAESAREAN SECTIONS AT THE INSTITUTO MATERNO-INFANTIL MARCH - AUGUST, 1981}

\section{SUMMARY}

This article summarizes the findings of a study on $\mathbf{5 4 9}$ medical records of patients with cesarian sections between March and August, 1981. There has been an increase of $12.6 \%$ in those operations.

Among the indications, fetal pelvic disproportion was pointed as the most usual $(29 \%)$, followed by iteration and toxemia in $4.7 \%$ of the cases.

The most frequent technique was a transperitoneum operation $(98 \%)$, and peridural anesthesia was used in $82 \%$ of the patients. These surgeries were

\section{BIBLIOGRAFIA}

1. GOODLIN, R: Aortocavl compression during cesarean seccion: A cause of newborn depression. Obst \& Gynecol 37 702, 1971.

2. PIVER, S. y Col: The safety of multiple cesarean section. Obst \& Gynecol 34: 690, 1969.

3. MICHAEL, J, y Col: The effect of fetal monitoring on the incidence of cesarean section. Obst \& Gynecol 49: 513, 1977.

4. HAGEN, D: Maternal febrile morbidity associated with fetal monitoring and cesarean section. Obst \& Gynecol 46: 26, 1975.

5. STEPHEN, L. y Col: Risk factors associated with post-cesarean section febrile morbidity. Obst \& Gynecol 49: 686, 1977. performed by surgeons in service training $(82 \%)$.

Most post-operatory complications were infeccions such as endometritis and wall cysts, but no relation was found between this pathology and ruptured membranes at admission. One woman died of sepsis. The average stay in the hospital was 5 days.

The majority of newborns weighed between 2.500 and 3.500 grams and had APGAR of 7-10. 23 newborns died and 17 cases of dead fetus during labor were recorded; in 19 cases, negative fetal signs at admission were the main indication for surgery.

6. CHOATE, J. y Col: Emergency cesarean section. Amer J. Obst \& Gynecol 100: 703, 1968,

7. EVRARD, J. y GOL, E.: Cesarean section and maternal mortality in Rhode Island. Amer J. Obst \& Gynecol 50: 594, 1977.

8. MANN, L. y Col: Modern indications for cesarean section. Amer J. Obst \& Gynecol 135: 437, 1979.

9. JONES, O: Cesarean section in present day obstetrics. Amer J. Obst \& Gynecol 125: 521, 1976.

10. WILLIAM: Obstetricia. Editorial Salvat, México, 1973.

11. LOPEZ, R: Morbilidad y mortalidad en el Instituto Materno Infantil Concepción Villaveces de Acosta. Rev. Col. de Obst. y Ginecol. 19, 1968. 
12. VALDERRAMA, D.: Anotaciones sobre cesáreas en hospitales y clínicas de Bogo. tá. Rev. Col. de Obst. y Ginecol. Vol. 16, No. 1, 1965.

13. DELGADO, C: Mortalidad perinatal en la operación cesárea. Rev. Col. de Obst. v Ginecol, 12, No, 5, 1961.

14. ACUÑA DIAZ J.G.: El parto vaginal en pacientes con antecedentes de cesárea. Rev. Col. de Obst y Ginecol. 15, No. 6, 1964.

15. ACUÑA DIAZ, J.G.: Parto expontáneo después de cesárea y cesárea a repetición. Rev. Col. de Obst. y Ginecol. 13, No. 1, 1962.

16. DEL CORRAL, F., MUÑOZ, S.: Evaluación clínica para el parto después de la cesárea. Rev. Col. de Obst. y Ginecol. 10, No. 1, 1959.

17. SANZ, M.: Conducta obstétrica en pacientes previamente operadas de cesárea. Rev. Col. de Obst. y Ginecol. Vol. 3, No. 2, 1952.
18. MARCUSHAMED, B. y Col: Morbimortalidad materno fetal en cesárea iterativa. Análisis de 1000 casos. Ginecol. y Obst de México 23: 613, 1968.

19. MILANES, J.; ZABALETA, A.: Cesárea extraperitoneal. Rev. Col. de Obst y Ginecol. 15, No. 4, 1964.

20. OFMAN, J.: Cesárea extraperitoneal por artificio. Rev. Col. de Obst. y Ginecol, 12, No. 6, 1956.

21. MILANES, J.; ZABALETA; SOTO: Cesárea Extraperitoneal. Rev. Col. de Obst. y Ginecol. 17: 425, 1966.

22. PERALTA, R.: Cesáreas previas y abortos, controversias clínicas y terapeúticas. 1962.

23. MUÑOZ, S. y COBO, E.: Indicaciones selectivas de la cesárea longitudinal. Rev. Col de Obst. y Ginecol. 11, 1960.

24. CARDENAS, A.; ZORNOSA DEL VALLE: La cesárea Iterativa. Revista Clínica Maternidad David Restrepo. Vol. 3. julio de 1962. 